\title{
Dispositivo automático para fabricar anillos de parafina utilizados en la industria textil
}

\section{(Automatic device to manufacture paraffin rings used in the Textile Industry)}

\author{
Luis Armando Hidalgo Aguilera, ${ }^{1}$ Santiago David Morejón Bravo ${ }^{1}$
}

\section{Resumen}

El trabajo consiste en realizar el diseño y la fabricación de un dispositivo automático que permita elaborar de manera continua anillos de parafina para que sean utilizados en los distintos procesos de la Industria Textil. La parafina es un subproducto del petróleo y es de mucha utilidad en la industria textil, puesto que contribuye a reducir la fricción entre los hilos y las diferentes guías por las que pasa, reduciendo la presencia de tensiones exageradas que provocan la rotura de los hilos, defectos en los tejidos y la paralización de las máquinas y de los procesos. El dispositivo, diseñado y validado en su estructura mecánica mediante el uso de un programa CAD, consta de un disco giratorio sobre el cual se encuentran instalados ocho moldes en forma de anillos de $54 \mathrm{~cm}^{3}$ de capacidad, espaciados y controlados automáticamente para ser llenados con parafina líquida de manera continua, garantizando la solidificación y extracción de cada anillo de manera sincronizada conforme el disco gira. El funcionamiento fue validado observando que los resultados de los ensayos de dosificación, tiempo de llenado, tiempo de traslado, tiempo de solidificación y tiempo de extracción del anillo solidificado contribuían a la productividad de este producto.

\section{Palabras clave}

Parafina; anillos; textiles; fricción; automático

\begin{abstract}
The work consists in the design and manufacture of an automatic device that allows to continuously elaborate paraffin rings used in the different processes of the Textile Industry. Paraffin is a byproduct of petroleum and is very useful in the Textile Industry, since it helps to reduce the friction between the threads and the different guides it goes through, reducing the presence of exaggerated tensions that cause the breakage of the threads, defects in the tissues and the paralysis of machines and processes. The device, designed and validated in its mechanical structure by means of the use of a CAD program, consists of a rotating disc on which eight molds, in the form of $54 \mathrm{~cm}^{3}$ capacity rings, are located, automatically spaced and controlled to be filled with paraffin liquid continuously, guaranteeing the solidification and extraction of each ring in a synchronized manner as the disc rotates. The performance was validated observing that the results of the tests of dosage, time of filling, time of transfer, solidification time and extraction time of the solidified ring, contributed to the productivity of this product.
\end{abstract}

\section{Keywords}

Paraffin; rings; textiles; friction; automatic

\section{Introducción}

La parafina es un producto ceroso derivado del petróleo, con cadenas de hidrocarburos rectas de longitud C18 hasta C40, sin ramificaciones, con estructura macrocristalina, de presentación sólida a temperatura ambiente, razón por la cual debe ser fundida para proceder con la elaboración de artículos en molde con este material, como es el caso de los anillos o aros de parafina (Kohl y otros, 2003) 
Los anillos de parafina son muy utilizados en la industria textil como recubrimiento de los hilos con el propósito de lubricarles y disminuir la fricción en los procesos complementarios de devanado y de tejido, mejorando de esta manera los índices de producción y calidad (López Guerra y Pacheco Carpio, 2017).

En el mundo es muy escaza la fabricación de máquinas destinadas a la producción específica de anillos de parafina, por lo que se adaptan con este propósito a ciertas máquinas diseñadas para la producción de velas u otro tipo de productos, ya que es alto el volumen de material que debe ser parafinado y elaborar manualmente estos anillos demanda gran inversión de tiempo y mano de obra.

En Ecuador, comercialmente no existen máquinas para la elaboración de anillos de parafina y el mercado textil se provee de este producto de dos maneras:

- Produciendo en forma manual, llenando moldes metálicos con parafina fundida, esperando un tiempo prudencial para que solidifiquen y finalmente extrayéndoles de los moldes. Este proceso toma mucho tiempo, encarece la mano de obra, no garantiza la calidad y en ocasiones no se logra cumplir con la demanda.

- Importando el producto desde Alemania, China, Colombia, Perú, entre otros. Este proceso eleva los costos de producción.

Con el objetivo de mejorar y asegurar la producción local de anillos de parafina para el uso textil, de manera cualitativa y cuantitativa, se propone el diseño e implementación de una máquina automática a un costo relativamente bajo respecto de lo que se podría con dificultad encontrar en el contexto mundial (Sánchez Silva, 2009).

El presente trabajo constituye una extensión del presentado en 2018 International Conference on Information Systems and Computer Science (INCISCOS) (Hidalgo y Morejón, 2018)

\section{Metodología}

Para el desarrollo del proyecto se utiliza la metodología de diseño mecatrónico basada en el modelo en V (Verenin, 2013), que determina las siguientes fases o etapas:

\subsection{Requerimientos del proyecto}

- Proceso continuo de elaboración de anillos de parafina.

- Materia prima: parafina en estado sólido.

- Sistema de fundición de la parafina.

- Dosificación automática de parafina fundida, por gravedad.

- Ocho moldes metálicos que produzcan anillos de: 40 mm de diámetro exterior, $15 \mathrm{~mm}$ de diámetro interior y $50 \mathrm{~mm}$ de altura máxima, con un volumen aproximado de $50 \mathrm{~cm}^{3}$ de parafina.

- Tiempo de solidificación, el comprendido entre la dosificación del primero y el último. A fin de garantizar la continuidad del proceso.

- Automática. 


\subsection{Diseño mecánico}

- Diseño geométrico y desarrollo del modelo en herramienta CAD.

- Análisis de cargas en la máquina y en cada componente.

- Diseño estructural de cada componente a construir.

- Selección de componentes existentes en el mercado.

- Ensamblaje de todos los componentes.

En la Figura 1 se muestra la ubicación de los principales componentes mecánicos cuya descripción se muestra en la Tabla 1.

Figura 1. Modelo del sistema mecánico

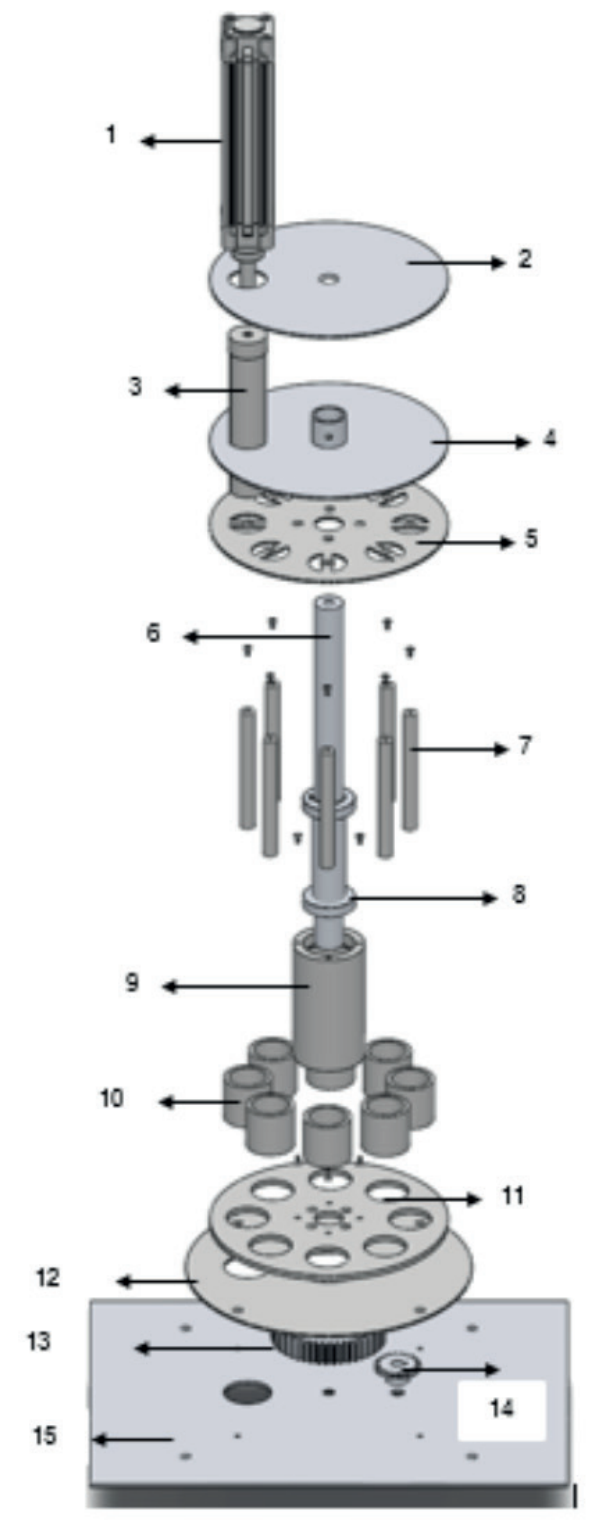




\subsection{Diseño eléctrico / electrónico}

- Selección del motor y elementos complementarios para su funcionamiento.

- Selección de electroválvulas para dosificación y extracción.

- Selección de un microcontrolador con una velocidad de respuesta apropiada entre la toma de datos y el control de los diferentes actuadores del sistema.

- Análisis y selección de sensores con capacidad de captar las señales que automaticen el sistema.

- Diseño de circuitos eléctricos / electrónicos.

\subsection{Diseño del control}

- Realizar el programa en un lenguaje compatible con el microcontrolador.

- Acondicionamiento de señales para control de actuadores de acuerdo con las señales recibidas por los sensores.

- Simulación del sistema de control.

\subsection{Implementación}

De acuerdo con la metodología usada, el proceso de implementación se realiza integrando los diferentes diseños de manera que no existan incompatibilidades tanto en el funcionamiento individual como en el sistema integrado, para lo cual se realizan verificaciones y ajustes permanentes durante el desarrollo del proyecto.

\subsection{Verificación y validación}

Una vez implementado el sistema automático para la elaboración de anillos de parafina se realizan las siguientes pruebas:

- Verificación de los desplazamientos de cada componente de acuerdo con la lógica de programación.

- Medición del tiempo de dosificación de la parafina fundida en los moldes.

- Medición del tiempo de solidificación de la parafina dosificada en los moldes.

- Medición de la capacidad de producción.

- Verificación de la calidad de los anillos de parafina obtenidos.

\section{Desarrollo del sistema}

El sistema funciona con un tipo de control de lazo abierto e inicia con la verificación de parte del operador de la existencia de parafina líquida y de aire comprimido a la presión de trabajo para arrancar con la producción automática una vez que los sensores transmitan la señal de que todos los elementos se encuentran en posición.

\subsection{Diseño mecánico}

Para el diseño y selección de los elementos del sistema mecánico, se asume un factor de diseño de 2.5 y se realiza un estudio de las cargas y esfuerzos a los que estarán sometidos cada 
uno, tomando en cuenta que ninguno de estos esfuerzos exceda a los permisibles del material, así como, que las deformaciones que produzcan las cargas no superen las permisibles para el correcto funcionamiento, mientras opera la máquina a plena carga. Este sistema constará básicamente de: estructura de acero inoxidable, ejes, tornillos, caja reductora, engranajes, cilindro neumático y válvula de paso, como se muestra en la Tabla 1.

Tabla 1. Componentes del Sistema Mecánico

\begin{tabular}{|l|l|}
\hline $\mathbf{n}^{{ }^{\circ}}$ & \\
\hline 11 & Cilindro neumático extractor \\
\hline 22 & Base extractor superior \\
\hline 33 & Extractor \\
\hline 44 & Base extractor inferior \\
\hline 55 & Base soporte tubos \\
\hline 66 & Eje central \\
\hline 77 & Tubos molde \\
\hline 88 & Rodamiento \\
\hline 09 & Bocín unión \\
\hline 110 & Moldes \\
\hline 111 & Base moldes \\
\hline 112 & Base perforada \\
\hline 113 & Engranaje de transmisión \\
\hline 114 & Piñón de transmisión \\
\hline 115 & Base máquina \\
\hline
\end{tabular}

Tanto para el modelado, como para la validación del diseño mecánico se realizan simulaciones con la ayuda de herramientas CAD (Gómez González, 2010), con el propósito de determinar el comportamiento de los esfuerzos, de las deformaciones y del factor de seguridad en cada uno de los elementos de la máquina.

\subsection{Cálculo estructural de las bases}

Como material se seleccionó el acero inoxidable AISI 304, cuyas propiedades mecánicas (IVAN BOHMAN) se muestran en la Tabla 2, y el cálculo consistió en seleccionar el espesor de la plancha, haciendo uso de las ecuaciones (1), (2), (3) y (4), según corresponda para cargas distribuidas o concentradas (Mott, 2017). Donde:

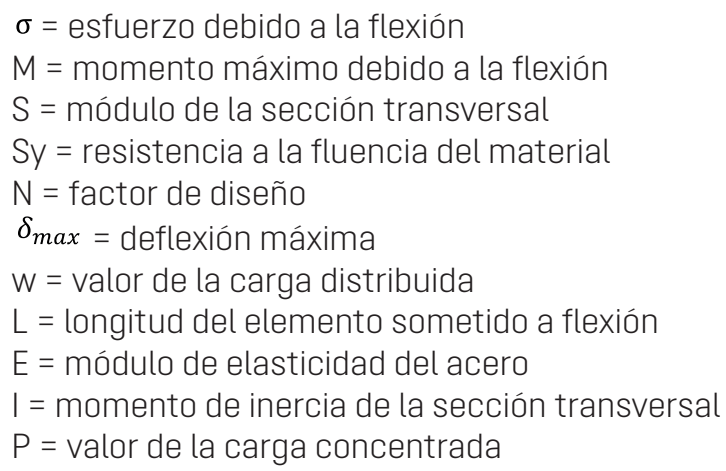


$a=$ distancia a la que se aplica la carga concentrada

$\mathrm{b}$ = valor de la base de la sección transversal

$\mathrm{h}$ = valor de la altura de la sección transversal

$\mathrm{h}=$ espesor de la plancha

$$
\begin{gathered}
\sigma=\frac{M}{S} \leq \frac{S_{y}}{N} \\
\delta_{\max }=-\frac{w L^{3}}{8 E I} \\
\delta_{\max }=-\frac{P a^{2}}{6 E I}(3 L-a) \\
I=\frac{b h^{3}}{12}
\end{gathered}
$$

Tabla 2. Propiedades mecánicas del acero inoxidable

\begin{tabular}{|l|l|}
\hline \multicolumn{1}{|c|}{ Propiedad } & \multicolumn{1}{c|}{ Valor } \\
\hline Módulo de elasticidad & $190 \mathrm{GPa}$ \\
\hline Sut & $586 \mathrm{MPa}$ \\
\hline Syt & $241 \mathrm{MPa}$ \\
\hline Peso específico & $7.93 \mathrm{gr} / \mathrm{cm}^{3}$ \\
\hline
\end{tabular}

\subsection{Diseño del eje central}

El eje central soporta el peso de todos los elementos del sistema mecánico y la fuerza de extracción que debe realizar el cilindro neumático, por lo que es diseñado considerando cargas combinadas y aplicando la ecuación (5), (Mott, 2017) en donde:

$M=$ momento flector resultante

$\mathrm{F}$ = fuerza axial resultante

A = área de la sección transversal del eje

$$
\sigma=\left(\frac{M}{S}+\frac{F}{A}\right) \leq \frac{S_{y}}{N}
$$

\subsection{Selección del cilindro neumático}

Para la selección del cilindro de doble efectos se determinó: que la fuerza necesaria para extraer el anillo de parafina fundido de su molde es de $10 \mathrm{~N}$, que la presión del aire en la red es de 4 bares y que la carrera del vástago debe ser de $160 \mathrm{~mm}$. Mediante el uso de la ecuación (6) (Creus Solé, 2011), se obtuvo el diámetro mínimo del cilindro.

$$
F_{C}=\frac{\pi D^{2}}{4} P R
$$


En donde:

$F_{C}=$ fuerza que debe ejercer el cilindro

$\mathrm{D}$ = diámetro del cilindro

$\mathrm{P}=$ presión del aire comprimido en la red

$\mathrm{R}=$ rendimiento del cilindro

\subsection{Diseño eléctrico / electrónico}

Se seleccionaron los componentes necesarios para la implementación del sistema de acuerdo con la potencia requerida para el funcionamiento de todos los actuadores eléctricos.

- Motorreductor trifásico con las características que se muestran en la Tabla 3.

Tabla 3. Especificaciones del motorreductor

\begin{tabular}{|l|l|}
\hline \multicolumn{1}{|c|}{ Potencia } & \multicolumn{1}{c|}{$\mathbf{1 1 0}$ watts } \\
\hline Voltaje & $220 / 380 / 440 \mathrm{VAC}$ \\
\hline Amperaje & $0.75 / 0.36 \mathrm{~A}$ \\
\hline Configuración & $\Delta / \mathrm{Y}$ \\
\hline rpm & $1350 / 22.5$ \\
\hline Torque & $16 \mathrm{Nm}$ \\
\hline Frecuencia & $60 \mathrm{~Hz}$ \\
\hline Factor de potencia & 0.71 \\
\hline
\end{tabular}

- Variador de frecuencia SINAMICS V20 INVERTER con las especificaciones que se muestra en la Tabla 4, para regular y controlar la velocidad del motorreductor (SIEMENS, 2016).

Tabla 4. Especificaciones del variador de frecuencia

\begin{tabular}{|l|l|}
\hline \multicolumn{1}{|c|}{ Voltaje de entrada } & \multicolumn{1}{|c|}{ Trifásico 400V AC, bifásico230V AC } \\
\hline Amperaje & $15-50 \mathrm{~A}$ \\
\hline Frecuencia & $47-60 \mathrm{~Hz}$ \\
\hline
\end{tabular}

- Microcontrolador Arduino MEGA

Las especificaciones para la selección del Microcontrolador se tomaron de (Arduino, 2018) considerando la propiedad de controlar puertos con salida PWM, debido a que el Arduino MEGA puede manejar a la vez, todos los elementos componentes del sistema.

Se diseñó el circuito de control para el variador de frecuencia y el motor que hace girar la base de los moldes. Figura 2. 
Figura 2. Circuito de control para el variador de frecuencia y el motor

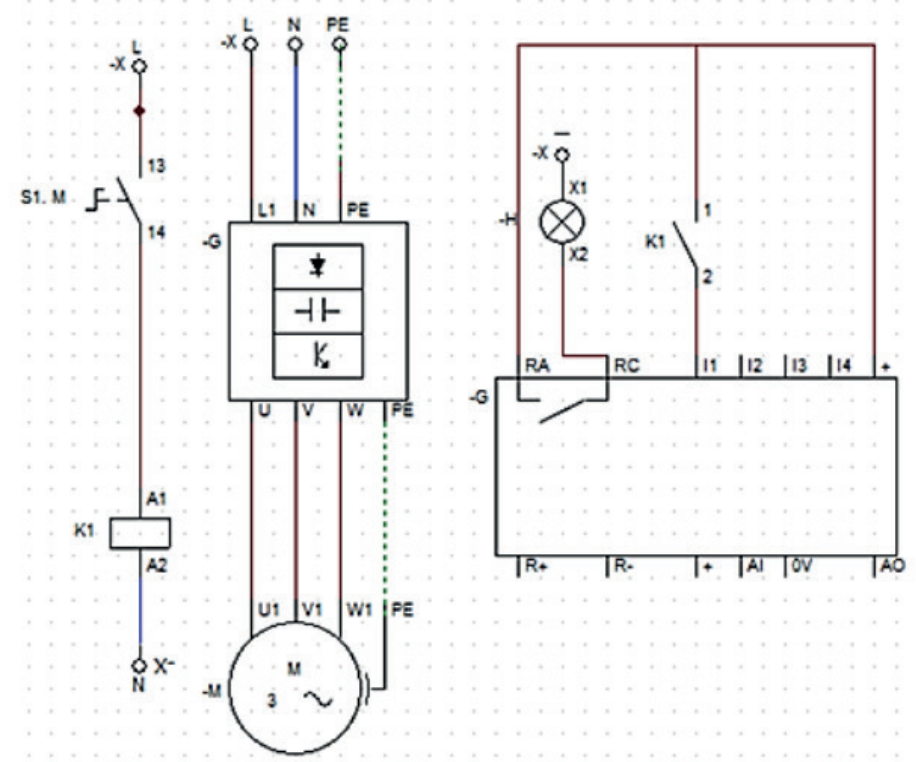

En la Figura 3, se muestra el circuito de control del cilindro neumático que hace trabajar al dosificador de parafina en los moldes.

Figura 3. Circuito de control para el cilindro neumático del dosificador

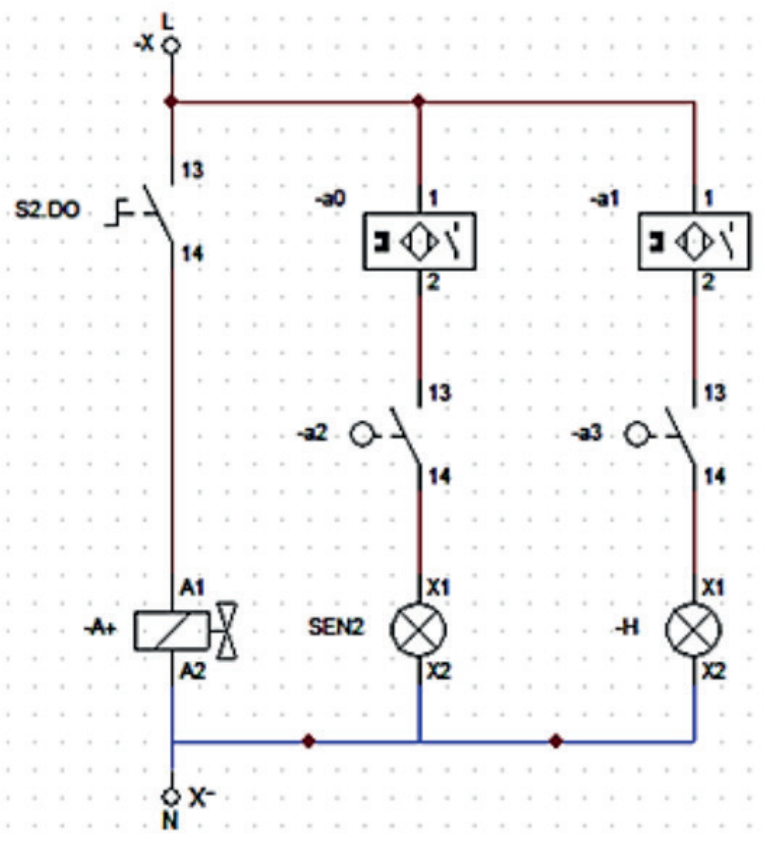

En la Figura 4, se muestra el circuito de control del cilindro neumático del extractor de los anillos de parafina ya solidificados. 
Figura 4. Circuito del cilindro neumático del extractor

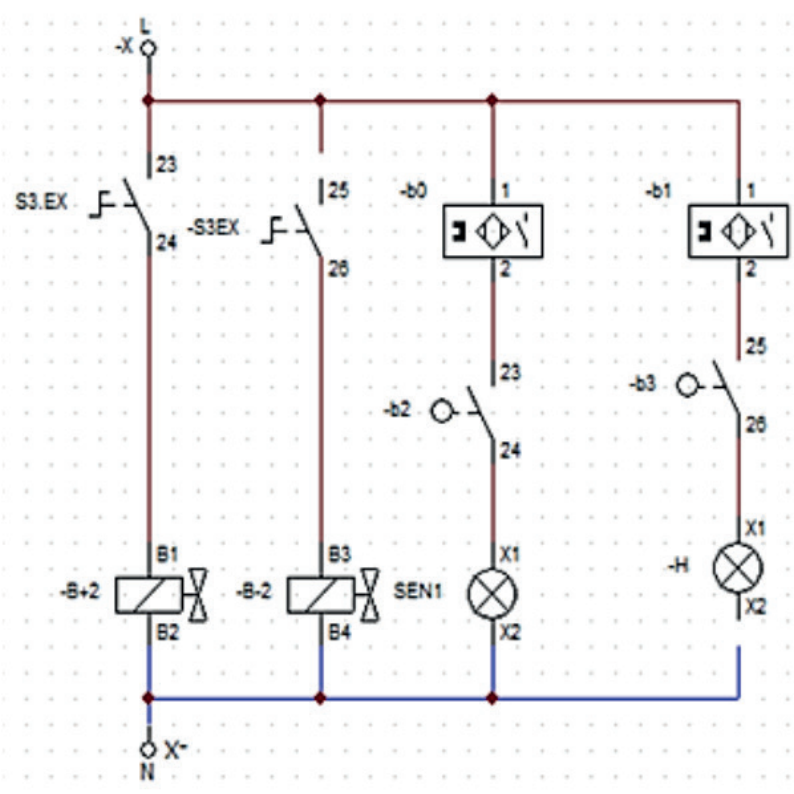

En la Figura 5, se muestran los mandos de los circuitos antes mencionados en las entradas y salidas que se usan en el microcontrolador Arduino Mega 2560.

Figura 5. Entradas y salidas conexión Arduino

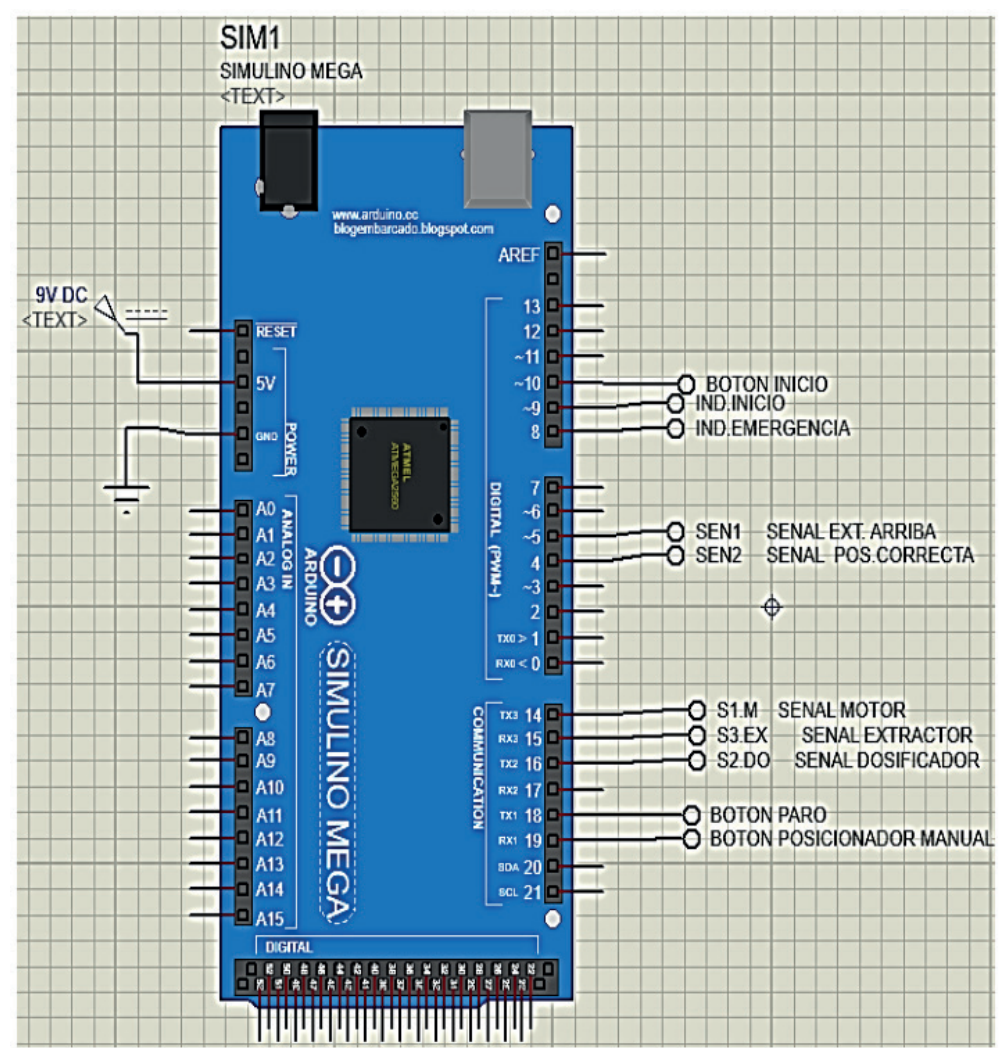




\subsection{Diseño de control del sistema}

La lógica de control del sistema se representa mediante el flujograma de la Figura 6, en la cual se muestran las condiciones y las acciones que se deben tomar en cuenta para que el proceso productivo se automático y continuo (Orna Chávez, Chuquín y Saquinga, 2018).

Como se mencionó anteriormente se seleccionó el Arduino Mega 2560 por su capacidad de manejo de puertos con salida PWM y por su sencillez y facilidad en la programación. Se controlan las dos electroválvulas que hacen funcionar a los cilindros neumáticos de dosificación y de extracción, así como también al motor que provoca el cambio de posición de los moldes.

Figura 6. Diagrama de flujo de lógica de control

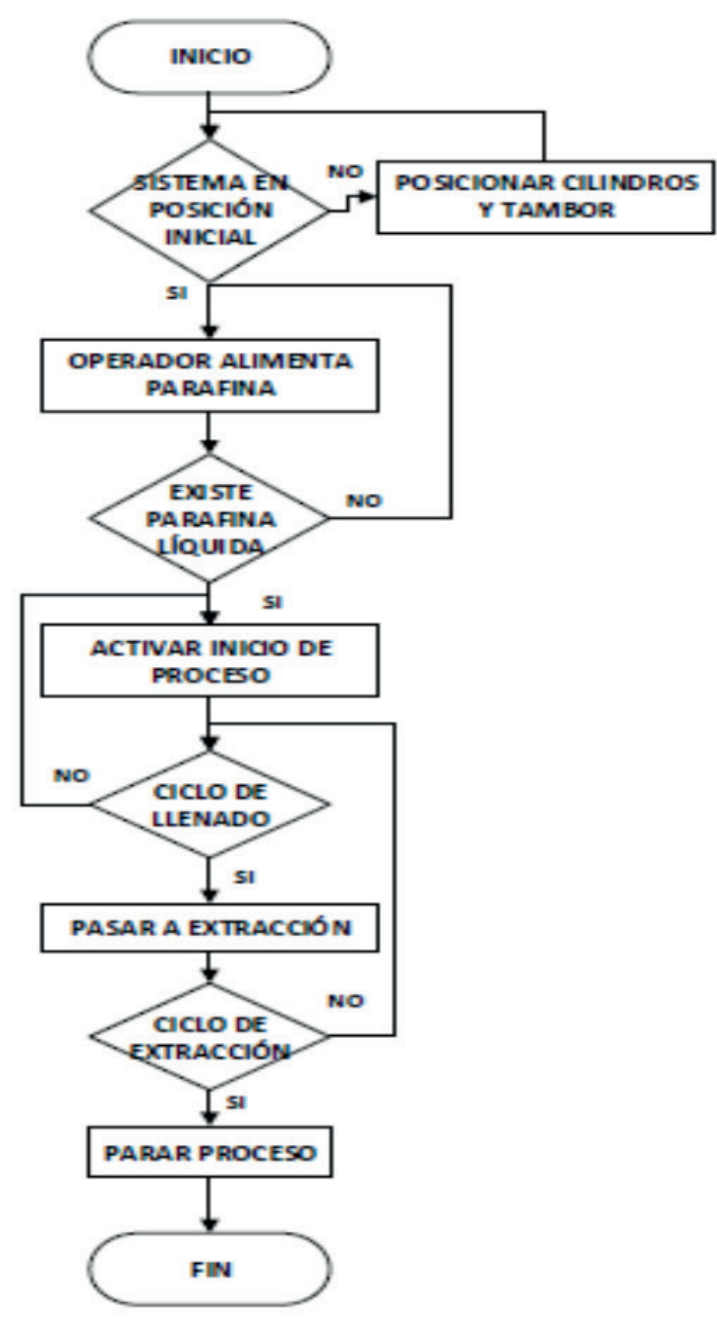

\section{Resultados}

En la Figura 7, se puede apreciar la implementación del dispositivo automático para elaborar anillos de parafina y en la Figura 8, los anillos obtenidos y la forma de usarlos en el devanado de hilo en las industrias textiles. 
Figura 7. Sistema automático para elaborar anillos de parafina
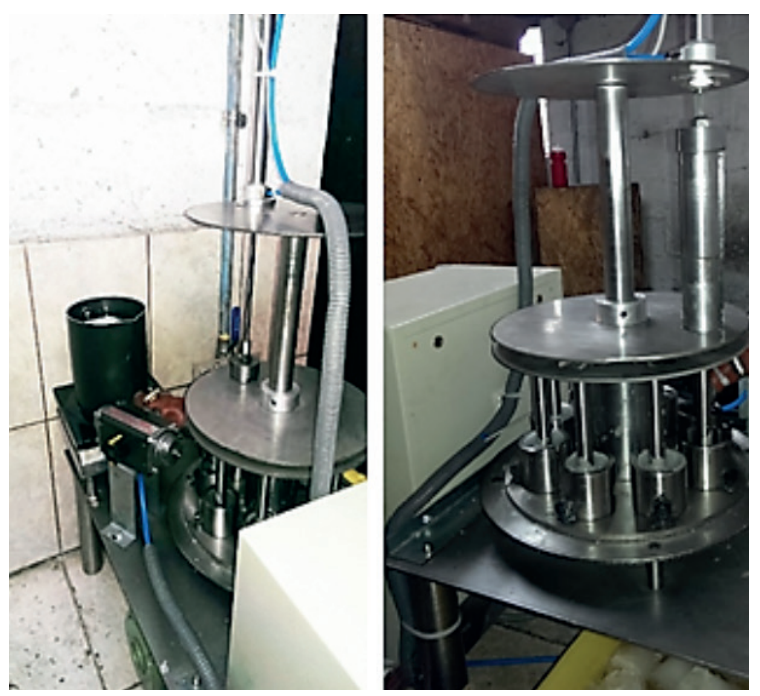

Figura 8. Anillos de parafina y su uso
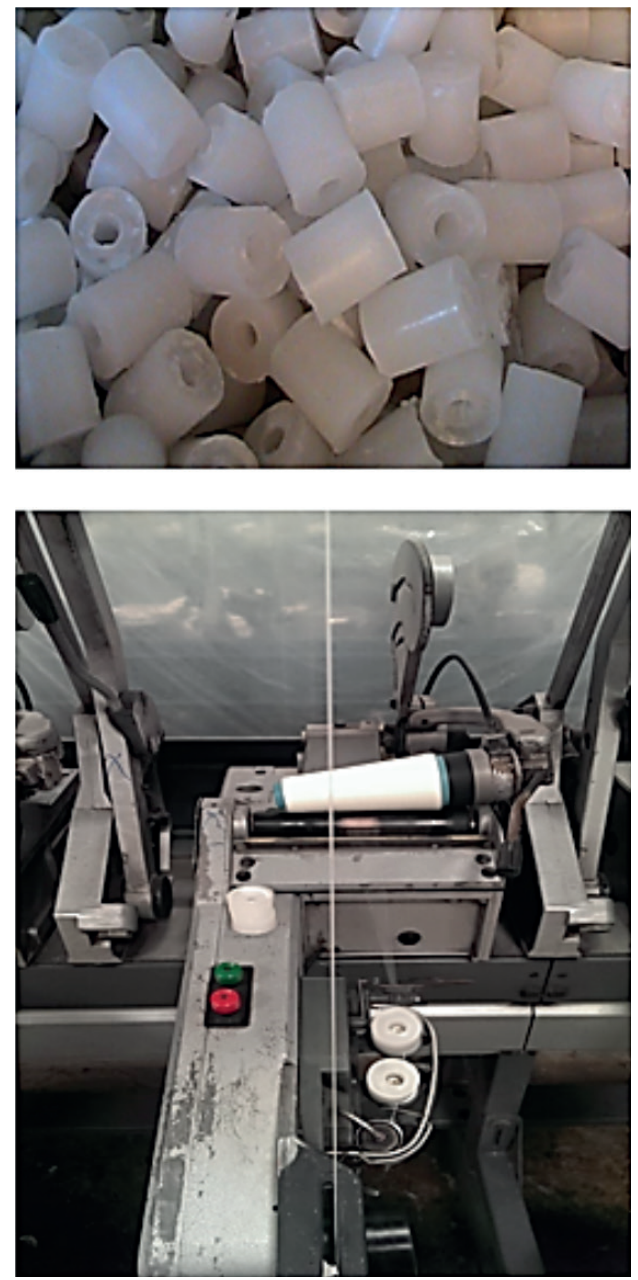
Con el propósito de calibrar y de determinar las ventajas del sistema implementado se realizaron las siguientes pruebas:

- Determinación del tiempo óptimo de dosificación de la parafina líquida en cada molde. Después de algunos ensayos se obtuvo que el tiempo exacto es de $0.6 \mathrm{~s}$ para lograr obtener anillos de $47 \mathrm{~mm}$ de altura promedio.

- Determinación del tiempo de solidificación de la parafina en cada molde. Luego de algunos ensayos y considerando un enfriamiento no forzado es decir sin ventilación adicional, se obtuvo que el tiempo necesario para una buena solidificación de la parafina en el molde es de 10 minutos, lo que significa que este es el tiempo que debe demorar el anillo desde que termina la dosificación hasta que se desplace al sitio de extracción.

- Determinación del tiempo de extracción del anillo de su molde. Este tiempo es una variable independiente ya que es el que se considere necesario para que el vástago se desplace hacia el anillo solidificado y lo extraiga del molde. Se lo fijo en $10 \mathrm{~s}$.

- Verificación de la calidad del producto. Una vez extraídos los anillos de parafina, se verificó que su acabado superficial sea estético, que exista simetría y que sus medidas cumplan con las especificaciones establecidas para este producto, obteniéndose la información que se muestra en la Tabla 5.

Tabla 5. Medidas obtenidas en los anillos de parafina

\begin{tabular}{|c|c|c|c|l|}
\hline $\mathbf{N}^{\circ}$ & $\begin{array}{c}\text { Diámetro exterior } \\
\mathbf{D} \\
{[\mathbf{m m}]}\end{array}$ & $\begin{array}{c}\text { Diámetro interior } \\
\mathbf{d} \\
{[\mathbf{m m}]}\end{array}$ & $\begin{array}{c}\text { Altura } \\
\mathbf{h} \\
{[\mathbf{m m}]}\end{array}$ & Estética y simetría \\
\hline 1 & 40 & 15 & 50 & Aceptable \\
\hline 2 & 40 & 15 & 48 & Aceptable \\
\hline 3 & 40 & 15 & 48 & Aceptable \\
\hline 4 & 40 & 15 & 47 & Aceptable \\
\hline 5 & 40 & 15 & 46 & Aceptable \\
\hline 6 & 40 & 15 & 45 & Aceptable \\
\hline 7 & 40 & 15 & 44 & Aceptable \\
\hline 8 & 40 & 15 & 44 & Aceptable \\
\hline
\end{tabular}

\section{Conclusiones y recomendaciones}

Se logra diseñar e implementar un sistema automático, que constituye una nueva propuesta para la elaboración de anillos de parafina, elementos de uso imprescindible en el devanado de hilos en la Industria Textil.

Tomando en cuenta que en la gran mayoría de las Industrias Textiles, estos anillos son elaborados en sus propias plantas de manera manual o en casos muy específicos los importan a precios relativamente altos, este sistema presenta las siguientes ventajas:

- Reduce el costo de mano de obra, puesto que el operador únicamente debe alimentar con parafina sólida y lubricar los moldes con aceite periódicamente, lo que le permite 
ocuparse en otras actividades o de ser necesario operar más de una máquina para la elaboración de anillos de parafina.

- Disminuye de manera considerable el desperdicio, ya que cada molde es dosificado con la cantidad exacta de parafina liquida, lo que además contribuye a obtener anillos uniformes en todas sus especificaciones.

- Reduce los riesgos, de sufrir accidentes o de adquirir algún tipo de lesión o enfermedad profesional, por la seguridad que se crea al tener un tipo de control amigable para el operador de la máquina.

Es un sistema innovador de fácil operación, automático y económico que puede ser replicado en cualquier empresa textil que requiera aumentar la producción de anillos de parafina de buena calidad, de una manera rápida, segura y económica.

Este sistema que consta de ocho moldes tiene una capacidad de producción de 480 anillos por día, aproximadamente 26 \% más que el proceso tradicional manual. Esta capacidad puede aumentar tan solamente aumentando el número de moldes, por lo que todo dependerá de las necesidades propias de cada empresa.

El sistema presenta gran facilidad para el montaje y desmontaje de cada uno de sus componentes, permitiendo de esta manera que la limpieza y el mantenimiento no requieran de actividades u operaciones complicadas.

Al iniciar el proceso se tiene los moldes fríos y la parafina líquida se solidifica con mayor facilidad es decir en menos tiempo, pero conforme transcurre el proceso, los moldes se calientan y este calentamiento del sistema puesto que todos los elementos son metálicos puede provocar que el tiempo de solidificación aumente, razón por la cual se recomienda de ser necesario adaptar un sistema de enfriamiento que mantenga constante el tiempo de solidificación de los anillos de parafina.

\section{Referencias}

Arduino. (2018). Arduino datasheet.

Creus Solé, A. (2011). Neumática e Hidráulica. CDMX, México: Alfaomega Grupo Editor, S. A. de C.V.

Gómez González, S. (2010). SolidWorks Simulation. CDMX, México: Alfaomega Grupo Editor, S. A. de C.V.

Hidalgo, L., y Morejón, S. (2018). Implementation of an Automatic System to Make Paraffin Rings. IEEEXplore Digital Library.

IVAN BOHMAN, I. (s. f.). Catálogo de Productos. Catálogo de Productos. Quito, Pichincha, Ecuador.

Kohl, R. A., Alcoba, M., Bruno, M., Varela, P., Cotella, N., y Radevich, O. (2003). Fundición de la cera perdida: Materiales para modelos, Jornadas SAM / Concamet / Simposio materio, 50-51NDICIÓN A LA CERA PERDIDA: MATERIALES PARA MODELOS. JORNADAS SAM/CONAMET/SIMPOSIO MATERIA, 50-51.

López Guerra, R. E., y Pacheco Carpio, R. (2017). Parafinado de Hilado para optimizar el proceso de tejido de punto en máquinas rectilíneas de prendas de fibra de alpaca. Mundo Textil 143, 15-19.

Mott, R. L. (2017). Machine Elements in Mechanical Desing. Dayton: Pearson.

Orna Chávez, J., Chuquín, N., y Saquinga, L. (2018). Diseño y construcción de una secadora automática para cacao a base de aire caliente tipo rotatorio para una capacidad de $500 \mathrm{~kg}$. Enfoque UTE, 159-174.

Sánchez Silva, M. L. (2009). Microencapsulación de materiales de cambio de fase para su aplicación textil. (Tesis de Doctorado). Universidad de Castilla La Mancha.

SIEMENS. (2016). Manual de especificaciones técnicas SINAMICS V2O INVERTER. NÜRNBERG.

Verenin, D. I. (2013). Design methodology for mechatronic systems. Düsseldorf. 